Bull. Austral. Math. Soc.

Vol. $71(2005)$ [435-446]

\title{
ON A SYSTEM OF \\ NONLINEAR PARTIAL DIFFERENTIAL EQUATIONS
}

\section{A. SANIH BONFOH}

We consider a generalised Cahn-Hilliard system with elasticity based on constitutives laws proposed by Gurtin, with a logarithmic free energy. We obtain some results on the existence and uniqueness of solutions.

\section{INTRODUCTION}

The Cahn-Hilliard equation is central to materials science. It is a conservation law (in the sense that the average of the order parameter is conserved) and describes very important qualitative features of two-phase systems, namely the transport of atoms between unit cells (see $[3,4]$ and the references therein). Some generalisations of this equation have been introduced by Gurtin in [5], which are based on constitutive equations that take into account the work of the internal microforces, the anisotropy and also the deformations of the material, which are essentially due to the displacement of atoms in the material. These derivations are based on belief that fundamental physical laws involving energy should account for the work associated with each kinematical process (the order parameter in our case). Assuming that the deformations are infinitesimal and that the displacement gradient is small, we can thus use the theory of linear elasticity. In this paper, we consider a model of these generalisations which have been derived in [7] and study the existence and uniqueness of solutions. system:

We set $\left.\Omega=\prod_{i=1}^{n}\right] 0, L_{i}\left[, \quad L_{i}>0, i=1, \ldots, n, n=2\right.$ or 3 , and consider the following

$$
\begin{gathered}
\frac{\partial \rho}{\partial t}-a . \nabla \frac{\partial \rho}{\partial t}=\operatorname{div}(B \nabla \mu) \\
\mu-b . \nabla \mu=-\alpha \Delta \rho+f^{\prime}(\rho)+\beta \frac{\partial \rho}{\partial t}-\frac{e}{2} \operatorname{TR}\left(C\left[\nabla u+{ }^{t} \nabla u\right]\right)+e^{2} \operatorname{TR}(\mathrm{CI})\left(\rho-\widetilde{\rho}_{0}\right) \\
\gamma \frac{\partial^{2} u}{\partial t^{2}}-\frac{1}{2} \operatorname{div}\left(C\left[\nabla u+{ }^{t} \nabla u\right]\right)+e \operatorname{div}(\rho \mathrm{CI})=0 \\
\left.\rho\right|_{t=0}=\rho_{0},\left.\quad u\right|_{t=0}=u_{0},\left.\quad \frac{\partial u}{\partial t}\right|_{t=0}=u_{1} \\
\rho \text { and } u \text { are } \Omega \text { - periodic; }
\end{gathered}
$$

Received June 8, 2005

Copyright Clearance Centre, Inc. Serial-fee code: 0004-9727/05 \$A2.00+0.00. 
where $\alpha, \beta, \gamma, e>0, \widetilde{\rho}_{0}$ is a constant, $a, b \in \mathbb{R}^{n}, B$ is a symmetric positive definite tensor with constant coefficients ( $B$ is called the mobility tensor), $C$ is the elasticity tensor with constant coefficients (we assume that it is a symmetric positive linear transformation which maps symmetric tensors onto symmetric tensors), $\mu$ is the chemical potential, $\rho$ is the order parameter (corresponding to a density of atoms) and $u$ is the displacement field. If $y$ is a motion of $\Omega$, then $y$ is a field that associates with each material point $x$ and time $t$ a point $y(x, t)=x+u(x, t)$. TR $(A)$ and ${ }^{t} A$ are the trace and the transpose of $A$ respectively. The free energy $f:[-1,1] \rightarrow \mathbb{R}$ is given by:

$$
\begin{aligned}
f(s) & \left.=\frac{1}{2}\left(1-s^{2}\right)+\frac{\theta}{2}\left[(1+s) \ln \left(\frac{1+s}{2}\right)+(1-s) \ln \left(\frac{1-s}{2}\right)\right], \quad s \in\right]-1,1[, \\
f(-1) & =f(1)=0,
\end{aligned}
$$

with $0<\theta<1$.

For the mathematical setting of the problem, we denote by $\|$.$\| and (.,$.$) the usual$ norm and scalar product in $L^{2}(\Omega)$ (which are extended to $\left.L^{2}(\Omega)^{n}\right)$. For each $\rho \in L^{1}(\Omega)$, $m(\rho)$ stands for the average of $\rho$, that is, $m(\rho)=(1 /|\Omega|) \int_{\Omega} \rho(x) d x$ (for a vector $u=\left(u_{1}, \ldots, u_{n}\right) \in L^{1}(\Omega)^{n}$, we have $\left.m(u)=\left(m\left(u_{1}\right), \ldots, m\left(u_{n}\right)\right) \in \mathbb{R}^{n}\right)$. For a space $\mathrm{X}$, we denote by $\dot{X}$ the space $\{q \in X, m(q)=0\}$, and by $X^{\prime}$ the dual space of $X$. We define by $N=-\operatorname{div} B \nabla$ a linear, self-adjoint, strictly positive operator with compact inverse $N^{-1}$ on $\dot{H}_{\text {per }}^{2}(\Omega)$. We set $\left.\Omega_{T}=\Omega \times\right] 0, T\left[\right.$ and $\bar{q}=q-m(q)$. We endow $\left(\dot{H}_{\text {per }}^{1}(\Omega)\right)^{\prime}$ with the norm $\|\cdot\|_{-1}$ defined by $\|\bar{q}\|_{-1}=\left\|N^{-1 / 2} \bar{q}\right\|, \forall q \in\left(H_{\text {per }}^{1}(\Omega)\right)^{\prime}$. Furthermore, there exist $c_{1}, c_{2}>0$ such that $\|\bar{q}\|_{-1} \leqslant c_{1}\|\bar{q}\| \leqslant c_{2}\|\nabla q\|, \forall q \in H_{\mathrm{per}}^{1}(\Omega)$. We finally note that $N$ and $\frac{\partial}{\partial x_{i}}$, and thus $N^{-1}$ and $\frac{\partial}{\partial x_{i}}, i=1, \ldots, n$, commute.

We introduce a weak formulation of the problem: Find

$$
(\rho, \mu, u):[0, T] \rightarrow H_{\mathrm{per}}^{1}(\Omega) \times H_{\mathrm{per}}^{1}(\Omega) \times \dot{H}_{\mathrm{per}}^{1}(\Omega)^{n}
$$

such that $\rho(0)=\rho_{0}, u(0)=u_{0}, \frac{\partial u}{\partial t}(0)=u_{1}$, and for almost everywhere $t \in[0, T], \forall T>0$,

$$
(\mu, q)+(\mu, b . \nabla q)=\alpha(\nabla \rho, \nabla q)+\left(f^{\prime}(\rho), q\right)+\beta\left(\frac{\partial \rho}{\partial t}, q\right)-\frac{e}{2}\left(\operatorname{TR}\left(C\left[\nabla u+{ }^{t} \nabla u\right]\right), q\right)
$$

$$
\left(\frac{\partial \rho}{\partial t}, q\right)+\left(\frac{\partial \rho}{\partial t}, a . \nabla q\right)=\left(N^{1 / 2} \mu, N^{1 / 2} q\right), \forall q \in H_{\mathrm{per}}^{1}(\Omega)
$$

$$
+e^{2} \mathrm{TR}(\mathrm{CI})\left(\rho-\tilde{\rho}_{0}, q\right), \forall q \in H_{\mathrm{per}}^{1}(\Omega) ;
$$

$$
\gamma\left(\frac{\partial^{2} u}{\partial t^{2}}, \eta\right)+\frac{1}{2}\left(C\left(\nabla u+{ }^{t} \nabla u\right), \nabla \eta\right)-e(\rho(\mathrm{CI}), \nabla \eta)=0, \quad \forall \eta \in \dot{H}_{\mathrm{per}}^{1}(\Omega)^{n}
$$

noting that $a . \nabla$ and $b . \nabla$ are antisymmetric on $H_{\text {per }}^{\mathrm{l}}(\Omega)$, that is, $(a . \nabla p, q)=-(p, a . \nabla q)$, $\forall p, q \in H_{\mathrm{per}}^{1}(\Omega)$. We take $q=1$ in (1.3) and observe that the average of $\rho$ is conserved:

$$
m(\rho(t))=m\left(\rho_{0}\right), \quad \forall t \geqslant 0 .
$$


We now take $q=1$ in (1.4) and obtain

$$
m(\mu)=m\left(f^{\prime}(\rho)\right)+e^{2} \mathrm{TR}(\mathrm{CI})\left(m(\rho)-\tilde{\rho_{0}}\right) .
$$

Setting $q=N^{-1} \bar{q}$ in (1.3), we get $\mu=-N^{-1} \frac{\partial \rho}{\partial t}+a . \nabla N^{-1} \frac{\partial \rho}{\partial t}+m(\mu)$; and substituting in (1.4) by standard computations and noting that

$$
b . \nabla\left(a . \nabla N^{-1} \frac{\partial \rho}{\partial t}\right)=\operatorname{div}\left(\left(a^{t} b+b^{t} a\right) / 2 \nabla N^{-1} \frac{\partial \rho}{\partial t}\right),
$$

we can reformulate problem (1.3)-(1.5) as follows:

We first look for $(\rho, u):[0, T] \rightarrow H_{\text {per }}^{1}(\Omega) \times \dot{H}_{\text {per }}^{1}(\Omega)^{n}$ such that

$$
\begin{aligned}
& \frac{d}{d t}\left[\left(N^{-1} \bar{\rho}, q\right)+\left(\widetilde{B} \nabla N^{-1} \bar{\rho}, \nabla q\right)+\left(N^{-1} \bar{\rho}, d . \nabla q\right)\right]+\alpha(\nabla \rho, \nabla q) \\
& -\frac{e}{2}\left(\operatorname{TR}\left(C\left[\nabla u+{ }^{t} \nabla u\right]\right), q\right)+e^{2} \operatorname{TR}(\mathrm{CI})\left(\rho-\widetilde{\rho}_{0}, \bar{q}\right)+\left(f^{\prime}(\rho), \bar{q}\right)=0, \\
& \forall q \in H_{\text {per }}^{1}(\Omega) \text {; } \\
& \gamma\left(\frac{\partial^{2} u}{\partial t^{2}}, \eta\right)+\frac{1}{2}\left(C\left(\nabla u+{ }^{t} \nabla u\right), \nabla \eta\right)-e(\rho(\mathrm{CI}), \nabla \eta)=0, \quad \forall \eta \in \dot{H}_{\mathrm{per}}^{1}(\Omega)^{n} ;
\end{aligned}
$$

and then set

$$
\mu=-N^{-1} \frac{\partial \rho}{\partial t}+a . \nabla N^{-1} \frac{\partial \rho}{\partial t}+m\left(f^{\prime}(\rho)\right)+e^{2} \mathrm{TR}(\mathrm{CI})\left(m(\rho)-\tilde{\rho_{0}}\right) ;
$$

where $d=a+b$ and $\widetilde{B}=\beta B-\left(a^{t} b+b^{t} a\right) / 2$. We proved in [2] that $\widetilde{B}$ is a positive tensor, thanks to thermodynamical considerations:

$$
\beta x^{2}+d \cdot y x+B y . y \geqslant 0, \forall x \in \mathbb{R}, \forall y \in \mathbb{R}^{n}
$$

(see [5]).

Throughout this paper, the same letter $c$ (and sometimes $c_{i}, i=0,1,2, \ldots$ ) shall denote positive constants that may change from line to line.

\section{A REGULARISED PROBLEM}

We denote by $\psi$ and $\phi$ the functions

$$
\psi(s)=\frac{\theta}{2}\left[(1+s) \ln \left(\frac{1+s}{2}\right)+(1-s) \ln \left(\frac{1-s}{2}\right)\right],
$$

and $\phi(s)=\psi^{\prime}(s)$, for $\left.s \in\right]-1,1\left[\right.$. We then have $f(s)=\left(1-s^{2}\right) / 2+\psi(s)$ and $f^{\prime}(s)=-s+\phi(s)$.

The major difficulty in the study of problem (1.3)-(1.5) is that $\phi(s)$ is singular at $s= \pm 1$ and, therefore, has no meaning if $\rho= \pm 1$ in an open set of non-zero measure. To overcome this difficulty, we consider a regularised problem as in [1]. The 
logarithmic free energy $f(\rho)$ is replaced by the twice continuously differentiable function $f_{\varepsilon}(s)=\left(1-s^{2}\right) / 2+\psi_{\varepsilon}(s)$, where $\left.\varepsilon \in\right] 0,1[$, and

$$
\psi_{\varepsilon}(s)= \begin{cases}\frac{\theta}{2}(1-s) \ln \left[\frac{1-s}{2}\right]+\frac{\theta}{4 \varepsilon}(1+s)^{2}+\frac{\theta}{2}(1+s) \ln \left[\frac{\varepsilon}{2}\right]-\frac{\theta \varepsilon}{4} & s \leqslant-1+\varepsilon \\ \psi(s) & |s| \leqslant 1-\varepsilon \\ \frac{\theta}{2}(1+s) \ln \left[\frac{1+s}{2}\right]+\frac{\theta}{4 \varepsilon}(1-s)^{2}+\frac{\theta}{2}(1-s) \ln \left[\frac{\varepsilon}{2}\right]-\frac{\theta \varepsilon}{4} & s \geqslant 1-\varepsilon\end{cases}
$$

The monotone function $\phi_{\varepsilon}=\psi_{\varepsilon}^{\prime}$ has the following properties (see [1]),

$$
\begin{gathered}
f_{\varepsilon}^{\prime}(s)(r-s) \leqslant f_{\varepsilon}(r)-f_{\varepsilon}(s)+\frac{1}{2}(r-s)^{2}, \\
\forall \varepsilon \leqslant \frac{1}{2}, \begin{cases}\theta(r-s)^{2} \leqslant\left(\phi_{\varepsilon}(r)-\phi_{\varepsilon}(s)\right)(r-s), & \forall r, s \\
\frac{\varepsilon}{\theta}\left(\phi_{\varepsilon}(r)-\phi_{\varepsilon}(s)\right)^{2} \leqslant\left(\phi_{\varepsilon}(r)-\phi_{\varepsilon}(s)\right)(r-s), & \forall r, s ;\end{cases}
\end{gathered}
$$

for $\varepsilon$ sufficiently small, for example, if $\varepsilon \leqslant \varepsilon_{0}=\theta / 8$, then

$$
f_{\varepsilon}(s) \geqslant \frac{\theta}{8 \varepsilon}\left([s-1]_{+}^{2}+[-1-s]_{+}^{2}\right)-1 \geqslant-1 \quad \forall s,
$$

where $[.]_{+}=\max \{, 0\}$.

We now study the corresponding regularised problem:

Find $\left(\rho_{\varepsilon}, \mu_{\varepsilon}, u_{\varepsilon}\right):[0, T] \rightarrow H_{\text {per }}^{1}(\Omega) \times H_{\text {per }}^{1}(\Omega) \times \dot{H}_{\text {per }}^{1}(\Omega)^{n}$, such that $\rho_{\varepsilon}(0)=\rho_{0}, u_{\varepsilon}(0)=u_{0}$, $\frac{\partial u_{\varepsilon}}{\partial t}(0)=u_{1}$, and for almost everywhere $t \in[0, T], \forall T>0$,

$$
\begin{aligned}
\frac{d}{d t}\left[\left(N^{-1} \bar{\rho}_{\varepsilon}, q\right)\right. & \left.+\left(\widetilde{B} \nabla N^{-1} \bar{\rho}_{\varepsilon}, \nabla q\right)+\left(N^{-1} \bar{\rho}_{\varepsilon}, d . \nabla q\right)\right]+\alpha\left(\nabla \rho_{\varepsilon}, \nabla q\right) \\
& -\frac{e}{2}\left(\operatorname{TR}\left(C\left[\nabla u_{\varepsilon}+{ }^{t} \nabla u_{\varepsilon}\right]\right), q\right)+e^{2} \operatorname{TR}(\mathrm{CI})\left(\rho_{\varepsilon}-\widetilde{\rho}_{0}, \bar{q}\right) \\
& +\left(f_{\varepsilon}^{\prime}\left(\rho_{\varepsilon}\right), \bar{q}\right)=0, \forall q \in H_{\mathrm{per}}^{1}(\Omega)
\end{aligned}
$$

$$
\gamma\left(\frac{\partial^{2} u_{\varepsilon}}{\partial t^{2}}, \eta\right)+\frac{1}{2}\left(C\left(\nabla u_{\varepsilon}+{ }^{t} \nabla u_{\varepsilon}\right), \nabla \eta\right)-e\left(\rho_{\varepsilon}(\mathrm{CI}), \nabla \eta\right)=0, \forall \eta \in \dot{H}_{\mathrm{per}}^{1}(\Omega)^{n}
$$

and

$$
\mu_{\varepsilon}=-N^{-1} \frac{\partial \rho_{\varepsilon}}{\partial t}+a \cdot \nabla N^{-1} \frac{\partial \rho_{\varepsilon}}{\partial t}+m\left(f_{\varepsilon}^{\prime}\left(\rho_{\varepsilon}\right)\right)+e^{2} \operatorname{TR}(\mathrm{CI})\left(m\left(\rho_{\varepsilon}\right)-\tilde{\rho}_{0}\right)
$$

LEMMA 2.1. We assume that $\left(\rho_{0}, u_{0}, u_{1}\right) \in H_{\mathrm{per}}^{1}(\Omega) \times \dot{H}_{\mathrm{per}}^{1}(\Omega)^{n} \times \dot{L}^{2}(\Omega)^{n}$, with $\left\|\rho_{0}\right\|_{L^{\infty}(\Omega)} \leqslant 1$, and that $\left.\left|m\left(\rho_{0}\right)\right| \leqslant 1-\delta, \delta \in\right] 0,1[$. Then, for all $\varepsilon$, there exists a unique trio of functions $\left(\rho_{\varepsilon}, \mu_{\varepsilon}, u_{\varepsilon}\right)$ solution of $(2.6)-(2.8)$ such that

$$
\rho_{\varepsilon} \in L^{\infty}\left(0, T ; H_{\mathrm{per}}^{1}(\Omega)\right) \cap L^{2}\left(0, T ; H_{\mathrm{per}}^{2}(\Omega)\right) \cap \mathcal{C}\left([0, T] ; L^{2}(\Omega)\right), \mu_{\varepsilon} \in L^{2}\left(0, T ; H_{\mathrm{per}}^{1}(\Omega)\right)
$$

and

$$
u_{\varepsilon} \in L^{\infty}\left(0, T ; \dot{H}_{\mathrm{per}}^{1}(\Omega)^{n}\right) \cap \mathcal{C}\left([0, T] ; \dot{L}^{2}(\Omega)^{n}\right)
$$


with $\frac{\partial \rho_{\varepsilon}}{\partial t} \in L^{2}\left(\Omega_{T}\right)$ and $\frac{\partial u_{\varepsilon}}{\partial t} \in L^{\infty}\left(0, T ; \dot{L}^{2}(\Omega)^{n}\right)$. Furthermore, we obtain uniform estimates with respect to $\varepsilon$, when $\varepsilon \leqslant \varepsilon_{0}$, for a sufficiently small $\varepsilon_{0}$.

Proof: (i) Existence. For a fixed $\varepsilon$, the existence of solution $\left(\rho_{\varepsilon}, \mu_{\varepsilon}\right)$ of $(2.6)-(2.7)$ follows from standard arguments using Galerkin approximations and then passing to the limit. In order to derive a priori estimates, we formally take $q=\frac{\partial \rho_{\varepsilon}}{\partial t}$ in (2.6). We note that

$$
\left(d . \nabla N^{-1} q, q\right)=0,\left(-\operatorname{div}\left(\widetilde{B} \nabla N^{-1} q\right), q\right)=\left\|\widetilde{B}^{1 / 2} \nabla B^{1 / 2} \nabla N^{-1} q\right\|^{2}, \forall q \in \dot{L}^{2}(\Omega) .
$$

Furthermore, the mapping $q \mapsto\left\|\widetilde{B}^{1 / 2} \nabla B^{1 / 2} \nabla N^{-1} q\right\|$ defines a norm in $\dot{L}^{2}(\Omega)$ that is equivalent to the usual $L^{2}(\Omega)$-norm (see [2]). Therefore,

$$
\begin{aligned}
& \frac{1}{2} \frac{d}{d t}\left(\alpha\left\|\nabla \rho_{\varepsilon}\right\|^{2}+e^{2} \operatorname{TR}(\mathrm{CI})\left\|\rho_{\varepsilon}\right\|^{2}+2 \int_{\Omega} f_{\varepsilon}\left(\rho_{\varepsilon}\right) d x\right) \\
& \quad+\left\|\widetilde{B}^{1 / 2} \nabla B^{1 / 2} \nabla N^{-1} \frac{\partial \rho_{\varepsilon}}{\partial t}\right\|^{2}+\left\|\frac{\partial \rho_{\varepsilon}}{\partial t}\right\|_{-1}^{2}-\frac{e}{2}\left(\operatorname{TR}\left[C\left(\nabla u_{\varepsilon}+{ }^{t} \nabla u_{\varepsilon}\right)\right], \frac{\partial \rho_{\varepsilon}}{\partial t}\right)=0
\end{aligned}
$$

and then

$$
\frac{d}{d t}\left(\alpha\left\|\nabla \rho_{\varepsilon}\right\|^{2}+e^{2} \mathrm{TR}(\mathrm{CI})\left\|\rho_{\varepsilon}\right\|^{2}+2 \int_{\Omega} f_{\varepsilon}\left(\rho_{\varepsilon}\right) d x\right)+c_{1}\left\|\frac{\partial \rho_{\varepsilon}}{\partial t}\right\|^{2} \leqslant c_{2}\left\|\nabla u_{\varepsilon}\right\|^{2} .
$$

We now take $\eta=u_{\varepsilon}$ in (2.7) and obtain

$$
\gamma\left(\frac{\partial^{2} u_{\varepsilon}}{\partial t^{2}}, u_{\varepsilon}\right)+\frac{1}{2}\left(C\left(\nabla u_{\varepsilon}+{ }^{t} \nabla u_{\varepsilon}\right), \nabla u_{\varepsilon}\right)=e\left(\rho_{\varepsilon}(\mathrm{CI}), \nabla u_{\varepsilon}\right) .
$$

We have $\left(C\left(\nabla u_{\varepsilon}+{ }^{t} \nabla u_{\varepsilon}\right), \nabla u_{\varepsilon}\right)=\left(C\left(\nabla u_{\varepsilon}+{ }^{t} \nabla u_{\varepsilon}\right), \nabla u_{\varepsilon}+{ }^{t} \nabla u_{\varepsilon}\right) / 2$ and thanks to Korn's inequality, there exists a positive constant $c_{0}$ such that

$$
\left(C\left(\nabla u_{\varepsilon}+{ }^{t} \nabla u_{\varepsilon}\right), \nabla u_{\varepsilon}\right) \geqslant c_{0}\left\|\nabla u_{\varepsilon}\right\|^{2}
$$

and therefore

$$
\gamma \frac{d}{d t}\left(\frac{\partial u_{\varepsilon}}{\partial t}, u_{\varepsilon}\right)+c_{1}\left\|\nabla u_{\varepsilon}\right\|^{2} \leqslant \gamma\left\|\frac{\partial u_{\varepsilon}}{\partial t}\right\|^{2}+c_{2}\left\|\rho_{\varepsilon}\right\|^{2} .
$$

We finally take $\eta=\frac{\partial u_{\varepsilon}}{\partial t}$ in (2.7) and obtain

$$
\frac{\gamma}{2} \frac{d}{d t}\left\|\frac{\partial u_{\varepsilon}}{\partial t}\right\|^{2}+\frac{1}{2}\left(C\left(\nabla u_{\varepsilon}+{ }^{t} \nabla u_{\varepsilon}\right), \nabla \frac{\partial u_{\varepsilon}}{\partial t}\right)=e\left(\rho_{\varepsilon}(\mathrm{CI}), \nabla \frac{\partial u_{\varepsilon}}{\partial t}\right)
$$

We have

$$
\begin{aligned}
\left(C\left(\nabla u_{\varepsilon}+{ }^{t} \nabla u_{\varepsilon}\right), \nabla \frac{\partial u_{\varepsilon}}{\partial t}\right) & =\frac{1}{2}\left(C\left(\nabla u_{\varepsilon}+{ }^{t} \nabla u_{\varepsilon}\right), \nabla \frac{\partial u_{\varepsilon}}{\partial t}+{ }^{t} \nabla \frac{\partial u_{\varepsilon}}{\partial t}\right) \\
& =\frac{1}{4} \frac{d}{d t}\left(C\left(\nabla u_{\varepsilon}+{ }^{t} \nabla u_{\varepsilon}\right), \nabla u_{\varepsilon}+{ }^{t} \nabla u_{\varepsilon}\right)
\end{aligned}
$$


and

$$
\left(\rho_{\varepsilon}(\mathrm{CI}), \nabla \frac{\partial u_{\varepsilon}}{\partial t}\right)=\frac{d}{d t}\left(\rho_{\varepsilon}(\mathrm{CI}), \nabla u_{\varepsilon}\right)-\left((\mathrm{CI}) \frac{\partial \rho_{\varepsilon}}{\partial t}, \nabla u_{\varepsilon}\right)
$$

Therefore,

$$
\begin{aligned}
\frac{d}{d t}\left(\gamma\left\|\frac{\partial u_{\varepsilon}}{\partial t}\right\|^{2}+\frac{1}{2}\left(C\left(\nabla u_{\varepsilon}+{ }^{t} \nabla u_{\varepsilon}\right), \nabla u_{\varepsilon}+{ }^{t} \nabla u_{\varepsilon}\right)-2 e\left(\rho_{\varepsilon}(\mathrm{CI}), \nabla u_{\varepsilon}\right)\right) \\
\leqslant \tau\left\|\frac{\partial \rho_{\varepsilon}}{\partial t}\right\|^{2}+c(\tau)\left\|\nabla u_{\varepsilon}\right\|^{2}
\end{aligned}
$$

for any positive real $\tau$.

We now add (2.10) and (2.17) with a proper $\tau$, and obtain an estimate of the form

$$
\frac{d E_{1}}{d t}+c_{1}\left\|\frac{\partial \rho_{\varepsilon}}{\partial t}\right\|^{2} \leqslant c_{2}\left\|\nabla u_{\varepsilon}\right\|^{2}
$$

where,

$$
\begin{aligned}
E_{1}(t)=\gamma\left\|\frac{\partial u_{\varepsilon}}{\partial t}\right\|^{2} & +\frac{1}{2}\left(C\left(\nabla u_{\varepsilon}+{ }^{t} \nabla u_{\varepsilon}\right), \nabla u_{\varepsilon}+{ }^{t} \nabla u_{\varepsilon}\right) \\
& -2 e\left(\rho_{\varepsilon}(\mathrm{CI}), \nabla u_{\varepsilon}\right)+\alpha\left\|\nabla \rho_{\varepsilon}\right\|^{2}+e^{2} \mathrm{TR}(\mathrm{CI})\left\|\rho_{\varepsilon}\right\|^{2}+2 \int_{\Omega} f_{\varepsilon}\left(\rho_{\varepsilon}\right) d x
\end{aligned}
$$

We now combine $\delta(2.13), \sigma(2.18)$ and $(2.10),(\delta, \sigma>0)$ and then obtain

$$
\frac{d E_{2}}{d t}+c_{1}\left\|\frac{\partial \rho_{\varepsilon}}{\partial t}\right\|^{2}+c_{2}\left\|\nabla u_{\varepsilon}\right\|^{2} \leqslant c_{3}\left(\left\|\nabla u_{\varepsilon}\right\|^{2}+\left\|\frac{\partial u_{\varepsilon}}{\partial t}\right\|^{2}+\left\|\rho_{\varepsilon}\right\|^{2}\right)
$$

where,

$$
\begin{aligned}
& E_{2}(t)=\gamma \delta\left(\frac{\partial u_{\varepsilon}}{\partial t}, u_{\varepsilon}\right)+\frac{\sigma}{2}\left(C\left(\nabla u_{\varepsilon}+{ }^{t} \nabla u_{\varepsilon}\right), \nabla u_{\varepsilon}+{ }^{t} \nabla u_{\varepsilon}\right) \\
& +\gamma \sigma\left\|\frac{\partial u_{\varepsilon}}{\partial t}\right\|^{2}-2 e \sigma\left(\rho_{\varepsilon}(\mathrm{CI}), \nabla u_{\varepsilon}\right)+\alpha(\sigma+1)\left\|\nabla \rho_{\varepsilon}\right\|^{2} \\
& +e^{2} \operatorname{TR}(\mathrm{CI})(\sigma+1)\left\|\rho_{\varepsilon}\right\|^{2}+2(\sigma+1) \int_{\Omega} f_{\varepsilon}\left(\rho_{\varepsilon}\right) d x .
\end{aligned}
$$

We first fix $\sigma$ such that

$$
\begin{aligned}
& \frac{\sigma}{4}\left(C\left(\nabla u_{\varepsilon}+{ }^{t} \nabla u_{\varepsilon}\right), \nabla u_{\varepsilon}+{ }^{t} \nabla u_{\varepsilon}\right)-2 e \sigma\left(\rho_{\varepsilon}(\mathrm{CI}), \nabla u_{\varepsilon}\right) \\
& +e^{2} \operatorname{TR}(\mathrm{CI})\left\|\rho_{\varepsilon}\right\|^{2} \geqslant c_{1}\left(\left\|\nabla u_{\varepsilon}\right\|^{2}+\left\|\rho_{\varepsilon}\right\|^{2}\right)
\end{aligned}
$$

where $c_{1}>0$; and we then fix $\delta$ such that

$$
\begin{aligned}
& \gamma \sigma\left\|\frac{\partial u_{\varepsilon}}{\partial t}\right\|^{2}+\frac{\sigma}{4}\left(C\left(\nabla u_{\varepsilon}+{ }^{t} \nabla u_{\varepsilon}\right), \nabla u_{\varepsilon}+{ }^{t} \nabla u_{\varepsilon}\right)+\gamma \delta\left(\frac{\partial u_{\varepsilon}}{\partial t}, u_{\varepsilon}\right) \\
& \geqslant c_{2}\left(\left\|\frac{\partial u_{\varepsilon}}{\partial t}\right\|^{2}+\left\|\nabla u_{\varepsilon}\right\|^{2}\right)
\end{aligned}
$$


where $c_{2}>0$. Estimates (2.22) and (2.23) come from the fact that, using Poincare and Young inequalities, we have

$$
-\left(\rho_{\varepsilon}(\mathrm{CI}), \nabla u_{\varepsilon}\right) \geqslant-\tau_{1} / 2\left\|\rho_{\varepsilon}\right\|^{2}-1 / 2 \tau_{1}\left\|\nabla u_{\varepsilon}\right\|^{2},
$$

and

$$
\left(\frac{\partial u_{\varepsilon}}{\partial t}, u_{\varepsilon}\right) \geqslant-\tau_{2} / 2\left\|\frac{\partial u_{\varepsilon}}{\partial t}\right\|^{2}-1 / 2 \tau_{2}\left\|\nabla u_{\varepsilon}\right\|^{2},
$$

for any strictly positive reals $\tau_{1}$ and $\tau_{2}$.

Thanks to $(2.21),(2.22)$ and (2.23), together with Poincaré's inequality, there exists $c_{\mathrm{f}}$ and $c_{2}$ such that

$$
E_{2}(t) \geqslant c_{1}\left(\left\|\frac{\partial u_{\varepsilon}}{\partial t}\right\|^{2}+\left\|\rho_{\varepsilon}\right\|_{H_{\text {per }}^{1}(\Omega)}^{2}+\left\|u_{\varepsilon}\right\|_{\dot{H}_{\text {per }}^{1}(\Omega)^{n}}^{2}+\int_{\Omega} f_{\varepsilon}\left(\rho_{\varepsilon}\right) d x\right)-c_{2} .
$$

We finally obtain from (2.20) an inequality of the form

$$
\frac{d E_{2}}{d t}+c_{1}\left\|\frac{\partial \rho_{\varepsilon}}{\partial t}\right\|^{2}+c_{2}\left\|u_{\varepsilon}\right\|_{\dot{H}_{\text {per }}^{1}(\Omega)^{n}}^{2} \leqslant c_{3} E_{2}+c_{4} \text {. }
$$

Using Gronwall's lemma and noting that $f_{\varepsilon}\left(\rho_{0}\right) \leqslant f\left(\rho_{0}\right)$ for $\varepsilon \leqslant \varepsilon_{0}, \varepsilon_{0}$ sufficiently small, we deduce the following estimates:

$$
\begin{array}{r}
\quad \underset{t \in[0, T]}{\operatorname{ess} \sup }\left\|\rho_{\varepsilon}\right\|_{H_{\mathrm{per}}^{1}(\Omega)}+\left\|\frac{\partial \rho_{\varepsilon}}{\partial t}\right\|_{L^{2}\left(\Omega_{T}\right)} \leqslant c ; \\
\underset{t \in[0, T]}{\operatorname{ess} \sup _{\Omega}} \int_{\Omega}\left(\left[\rho_{\varepsilon}-1\right]_{+}^{2}+\left[-1-\rho_{\varepsilon}\right]_{+}^{2}\right) d x \leqslant c \varepsilon ; \\
\underset{t \in[0, T]}{\operatorname{ess} \sup }\left\|\frac{\partial u_{\varepsilon}}{\partial t}\right\|+\underset{t \in[0, T]}{\operatorname{ess} \sup }\left\|u_{\varepsilon}\right\|_{\dot{H}_{\text {per }}^{1}(\Omega)^{\mathrm{n}}} \leqslant c ;
\end{array}
$$

where $c$ is independent of $\varepsilon$. The existence of $\rho_{\varepsilon}$ and $u_{\varepsilon}$ are deduced from (2.26) and (2.28) using classical compactness results (see for instance [6] or [8]). The existence of $\mu_{\varepsilon}$ is deduced from (2.8).

We further obtain some uniform estimates in $\varepsilon$, for $\varepsilon \leqslant \varepsilon_{0}, \varepsilon_{0}$ sufficiently small. We note that

$$
\left\|\nabla N^{-1} \frac{\partial \rho_{\varepsilon}}{\partial t}\right\| \leqslant c\left\|\frac{\partial \rho_{\varepsilon}}{\partial t}\right\|_{-1} \leqslant c\left\|\frac{\partial \rho_{\varepsilon}}{\partial t}\right\|
$$

and

$$
\left\|\nabla\left(a . \nabla N^{-1} \frac{\partial \rho_{\varepsilon}}{\partial t}\right)\right\| \leqslant c\left\|\frac{\partial \rho_{\varepsilon}}{\partial t}\right\|
$$

and therefore

$$
\left\|\nabla \mu_{\varepsilon}\right\| \leqslant c\left\|\frac{\partial \rho_{\varepsilon}}{\partial t}\right\|
$$

and thanks to Poincaré's inequality and the regularised counterparts of (1.6) and (1.7), we obtain

$$
\left\|\mu_{\varepsilon}\right\|_{L^{2}\left(0, T ; H_{\mathrm{per}}^{1}(\Omega)\right)}^{2} \leqslant c\left(1+\left\|m\left(f_{\varepsilon}^{\prime}\left(\rho_{\varepsilon}\right)\right)\right\|_{L^{2}\left(\Omega_{T}\right)}^{2}\right)
$$


We now want to prove that $\left\|m\left(f_{\varepsilon}^{\prime}\left(\rho_{\varepsilon}\right)\right)\right\|_{L^{2}\left(\Omega_{T}\right)}^{2}$ is bounded independently of $\varepsilon$.

We formally take $q=\bar{\rho}_{\varepsilon}$ in the regularised counterpart of (1.4), and obtain

$$
\begin{array}{r}
\alpha\left\|\nabla \rho_{\varepsilon}\right\|^{2}+\left(f_{\varepsilon}^{\prime}\left(\rho_{\varepsilon}\right), \bar{\rho}_{\varepsilon}\right)=\left(\mu_{\varepsilon}-b . \nabla \mu_{\varepsilon}-\beta \frac{\partial \rho_{\varepsilon}}{\partial t}+\frac{e}{2} \operatorname{TR}\left(C\left[\nabla u_{\varepsilon}+{ }^{t} \nabla u_{\varepsilon}\right]\right)\right. \\
\left.-e^{2} \operatorname{TR}(\mathrm{CI})\left(\rho_{\varepsilon}-\widetilde{\rho}_{0}\right), \bar{\rho}_{\varepsilon}\right)
\end{array}
$$

Noting that

$$
\left(f_{\varepsilon}^{\prime}\left(\rho_{\varepsilon}\right), \bar{\rho}_{\varepsilon}\right)=\left(f_{\varepsilon}^{\prime}\left(\rho_{\varepsilon}\right), \rho_{\varepsilon}-\lambda\right)+\left(f_{\varepsilon}^{\prime}\left(\rho_{\varepsilon}\right), \lambda-m\left(\rho_{\varepsilon}\right)\right), \quad \forall \lambda \in \mathbb{R},
$$

and using (2.3), it follows that

$$
\begin{aligned}
\left(f_{\varepsilon}^{\prime}\left(\rho_{\varepsilon}\right), \lambda-m\left(\rho_{\varepsilon}\right)\right) \leqslant\left(f_{\varepsilon}(\lambda)-\right. & \left.f_{\varepsilon}\left(\rho_{\varepsilon}\right), 1\right)+\frac{1}{2}\left\|\rho_{\varepsilon}-\lambda\right\|^{2}+c_{1}\left\|\nabla \rho_{\varepsilon}\right\|^{2} \\
& +c_{2}\left(\left\|\mu_{\varepsilon}\right\|+\left\|\nabla \mu_{\varepsilon}\right\|+\left\|\nabla u_{\varepsilon}\right\|+\left\|\frac{\partial \rho_{\varepsilon}}{\partial t}\right\|\right)\left\|\nabla \rho_{\varepsilon}\right\| .
\end{aligned}
$$

Choosing $\lambda= \pm 1$ and using (2.5) and the assumptions on $\rho_{0}$, we deduce

$$
\delta|\Omega|\left|m\left(f_{\varepsilon}^{\prime}\left(\rho_{\varepsilon}\right)\right)\right| \leqslant c\left[1+\left\|\nabla \rho_{\varepsilon}\right\|^{2}+\left(\left\|\nabla \mu_{\varepsilon}\right\|+\left\|\nabla u_{\varepsilon}\right\|+\left\|\frac{\partial \rho_{\varepsilon}}{\partial t}\right\|\right)\left\|\nabla \rho_{\varepsilon}\right\|\right],
$$

and, therefore,

$$
\left\|m\left(f_{\varepsilon}^{\prime}\left(\rho_{\varepsilon}\right)\right)\right\|^{2} \leqslant c\left[1+\left\|\nabla \rho_{\varepsilon}\right\|^{4}+\left(\left\|\nabla \mu_{\varepsilon}\right\|^{2}+\left\|\nabla u_{\varepsilon}\right\|^{2}+\left\|\frac{\partial \rho_{\varepsilon}}{\partial t}\right\|^{2}\right)\left\|\nabla \rho_{\varepsilon}\right\|^{2}\right]
$$

hence

$$
\left\|m\left(f_{\varepsilon}^{\prime}\left(\rho_{\varepsilon}\right)\right)\right\|_{L^{2}\left(\Omega_{T}\right)}^{2} \leqslant c
$$

Taking now $q=\phi_{\varepsilon}\left(\rho_{\varepsilon}\right)-m\left(\phi_{\varepsilon}\left(\rho_{\varepsilon}\right)\right)$ in the regularised counterpart of (1.4), we obtain

$$
\begin{aligned}
\alpha\left(\phi_{\varepsilon}^{\prime}\left(\rho_{\varepsilon}\right) \nabla \rho_{\varepsilon},\right. & \left.\nabla \rho_{\varepsilon}\right)+\left(\phi_{\varepsilon}\left(\rho_{\varepsilon}\right)-\rho_{\varepsilon}, \phi_{\varepsilon}\left(\rho_{\varepsilon}\right)-m\left(\phi_{\varepsilon}\left(\rho_{\varepsilon}\right)\right)\right) \\
= & \left(\mu_{\varepsilon}-b . \nabla \mu_{\varepsilon}-\beta \frac{\partial \rho_{\varepsilon}}{\partial t}+\frac{e}{2} \operatorname{TR}\left(C\left[\nabla u+{ }^{t} \nabla u\right]\right)\right. \\
& \left.-e^{2} \operatorname{TR}(\mathrm{CI})\left(\rho_{\varepsilon}-\tilde{\rho}_{0}\right), \phi_{\varepsilon}\left(\rho_{\varepsilon}\right)-m\left(\phi_{\varepsilon}\left(\rho_{\varepsilon}\right)\right)\right) .
\end{aligned}
$$

We note that $\phi_{\varepsilon}^{\prime}\left(\rho_{\varepsilon}\right) \geqslant \theta$ (which follows from (2.4)), and then deduce the following estimate

$$
\begin{aligned}
\alpha \theta\left\|\nabla \rho_{\varepsilon}\right\|^{2}+\frac{1}{2}\left\|\phi_{\varepsilon}\left(\rho_{\varepsilon}\right)-m\left(\phi_{\varepsilon}\left(\rho_{\varepsilon}\right)\right)\right\|^{2} & \leqslant c_{1}\left(1+\left\|\mu_{\varepsilon}\right\|^{2}\right. \\
& \left.+\left\|\nabla \mu_{\varepsilon}\right\|^{2}+\left\|\nabla \rho_{\varepsilon}\right\|^{2}+\left\|\nabla u_{\varepsilon}\right\|^{2}+\left\|\frac{\partial \rho_{\varepsilon}}{\partial t}\right\|^{2}\right)
\end{aligned}
$$


which yields $\left\|\phi_{\varepsilon}\left(\rho_{\varepsilon}\right)-m\left(\phi_{\varepsilon}\left(\rho_{\varepsilon}\right)\right)\right\|_{L^{2}\left(\Omega_{T}\right)}^{2} \leqslant c$ and, therefore, $\left\|\phi_{\varepsilon}\left(\rho_{\varepsilon}\right)\right\|_{L^{2}\left(\Omega_{T}\right)} \leqslant c$.

Finally, the uniform estimate on $\rho_{\varepsilon}$ in $L^{2}\left(0, T ; H_{\mathrm{per}}^{2}(\Omega)\right)$ follows from the estimate

$$
\begin{aligned}
\left\|\Delta \rho_{\varepsilon}\right\|_{L^{2}\left(\Omega_{T}\right)}^{2} \leqslant c_{1}\left(1+\left\|\mu_{\varepsilon}\right\|_{L^{2}\left(\Omega_{T}\right)}^{2}+\| \nabla\right. & \mu_{\varepsilon}\left\|_{L^{2}\left(\Omega_{T}\right)}^{2}+\right\| \nabla u_{\varepsilon} \|_{L^{2}\left(\Omega_{T}\right)}^{2} \\
& \left.+\left\|\phi_{\varepsilon}\left(\rho_{\varepsilon}\right)\right\|_{L^{2}\left(\Omega_{T}\right)}^{2}+\left\|\frac{\partial \rho_{\varepsilon}}{\partial t}\right\|_{L^{2}\left(\Omega_{T}\right)}^{2}\right) \leqslant c_{2} ;
\end{aligned}
$$

which follows from the second line of the regularised counterpart of (1.1) and the fact that the following regular inequality

$$
\left\|\rho_{\varepsilon}-m\left(\rho_{0}\right)\right\|_{L^{2}\left(0, T ; H_{\text {per }}^{2}(\Omega)\right)}^{2} \leqslant c\left\|\Delta \rho_{\varepsilon}\right\|_{L^{2}\left(\Omega_{T}\right)}^{2}
$$

is held.

(ii) Uniqueness. Let $\left(\rho_{1}, \mu_{1}, u_{1}\right)$ and $\left(\rho_{2}, \mu_{2}, u_{2}\right)$ be two solutions of $(2.6)-(2.8)$ with the same initial data. Setting $\rho=\rho_{1}-\rho_{2}$ and $u=u_{1}-u_{2}$, we have $\rho(0)=0, u(0)=0$, $\frac{\partial u}{\partial t}(0)=0$ and

$$
\begin{aligned}
\frac{d}{d t}\left[\left(N^{-1} \rho, q\right)+\right. & \left.\left(\widetilde{B} \nabla N^{-1} \rho, \nabla q\right)+\left(N^{-1} \rho, d . \nabla q\right)\right]+\alpha(\nabla \rho, \nabla q) \\
& -\frac{e}{2}\left(\operatorname{TR}\left(C\left[\nabla u+{ }^{t} \nabla u\right]\right), q\right)+e^{2} \operatorname{TR}(\mathrm{CI})(\rho, \bar{q}) \\
& +\left(f_{\varepsilon}^{\prime}\left(\rho_{1}\right)-f_{\varepsilon}^{\prime}\left(\rho_{2}\right), \bar{q}\right)=0, \forall q \in H_{\mathrm{per}}^{1}(\Omega)
\end{aligned}
$$

$$
\gamma\left(\frac{\partial^{2} u}{\partial t^{2}}, \eta\right)+\frac{1}{2}\left(C\left(\nabla u+{ }^{t} \nabla u\right), \nabla \eta\right)-e(\rho(\mathrm{CI}), \nabla \eta)=0, \quad \forall \eta \in \dot{H}_{\text {per }}^{1}(\Omega)^{n} .
$$

We take $q=\rho$ in (2.39), and noting that $m(\rho)=0$, we obtain

$$
\begin{aligned}
\frac{d}{d t}\left[\| \widetilde{B}^{1 / 2} \nabla\right. & \left.B^{1 / 2} \nabla N^{-1} \rho\left\|^{2}+\right\| \rho \|_{-1}^{2}\right]+\alpha\|\nabla \rho\|^{2}-\frac{e}{2}\left(\operatorname{TR}\left(C\left[\nabla u+{ }^{t} \nabla u\right]\right), \rho\right) \\
& +e^{2} \operatorname{TR}(\mathrm{CI})\|\rho\|^{2}+\left(\phi_{\varepsilon}\left(\rho_{1}\right)-\phi_{\varepsilon}\left(\rho_{2}\right), \rho\right)=\|\rho\|^{2}-\left(\frac{\partial \rho}{\partial t}, d . \nabla N^{-1} \rho\right) .
\end{aligned}
$$

We have $\left(\phi_{\varepsilon}\left(\rho_{1}\right)-\phi_{\varepsilon}\left(\rho_{2}\right), \rho\right) \geqslant \theta\|\rho\|^{2}$ (which comes from (2.4)) and $\|\rho\|_{H_{\text {per }}^{1}(\Omega)} \leqslant c\|\nabla \rho\|$ (Poincaré's inequality). Therefore,

$$
\frac{d}{d t}\left(\left\|\widetilde{B}^{1 / 2} \nabla B^{1 / 2} \nabla N^{-1} \rho\right\|^{2}+\|\rho\|_{-1}^{2}\right)+c_{1}\|\rho\|_{H_{\text {per }}^{1}(\Omega)}^{2} \leqslant c_{2}\left(\|\rho\|^{2}+\|\nabla u\|^{2}+\left\|\frac{\partial \rho}{\partial t}\right\|^{2}\right)
$$

We now take $q=\frac{\partial \rho}{\partial t}$ in (2.39) and obtain

$$
\frac{1}{2} \frac{d}{d t}\left(\alpha\|\nabla \rho\|^{2}+e^{2} \mathrm{TR}(\mathrm{CI})\|\rho\|^{2}\right)+\left\|\widetilde{B}^{1 / 2} \nabla B^{1 / 2} \nabla N^{-1} \frac{\partial \rho}{\partial t}\right\|^{2}+\left\|\frac{\partial \rho}{\partial t}\right\|_{-1}^{2}
$$

$$
-\frac{e}{2}\left(\operatorname{TR}\left[C\left(\nabla u+{ }^{t} \nabla u\right)\right], \frac{\partial \rho}{\partial t}\right)+\left(\phi_{\varepsilon}\left(\rho_{1}\right)-\phi_{\varepsilon}\left(\rho_{2}\right), \frac{\partial \rho}{\partial t}\right)=\left(\rho, \frac{\partial \rho}{\partial t}\right) .
$$


We note that $\phi_{\varepsilon}\left(\rho_{1}\right)-\phi_{\varepsilon}\left(\rho_{2}\right)=\rho \phi_{\varepsilon}^{\prime}\left(\zeta \rho_{1}+(1-\zeta) \rho_{2}\right)$, with $\zeta \in[0,1]$; and that $\phi_{\varepsilon}^{\prime}(s) \leqslant \theta \varepsilon^{-1}, \forall s$, (which follows from (2.4)). We thus have

$$
\left(\phi_{\varepsilon}\left(\rho_{1}\right)-\phi_{\varepsilon}\left(\rho_{2}\right), \frac{\partial \rho}{\partial t}\right) \leqslant \eta\left\|\frac{\partial \rho}{\partial t}\right\|^{2}+c \varepsilon^{-2}\|\rho\|, \forall \eta>0,
$$

and

$$
\frac{d}{d t}\left(\alpha\|\nabla \rho\|^{2}+e^{2} \operatorname{TR}(\mathrm{CI})\|\rho\|^{2}\right)+c_{1}\left\|\frac{\partial \rho}{\partial t}\right\|^{2} \leqslant c_{2}\left(1+\varepsilon^{-2}\right)\|\rho\|^{2}+c_{3}\|\nabla u\|^{2} .
$$

We combine $\sigma(2.42)$ and (2.44) with a proper positive $\sigma$ and obtain

$$
\begin{aligned}
\frac{d}{d t}\left[\sigma\left\|\tilde{B}^{1 / 2} \nabla B^{1 / 2} \nabla N^{-1} \rho\right\|^{2}\right. & \left.+\sigma\|\rho\|_{-1}^{2}+\alpha\|\nabla \rho\|^{2}+e^{2} \operatorname{TR}(\mathrm{CI})\|\rho\|^{2}\right] \\
& +c_{1}\|\rho\|_{H_{\text {per }}^{1}(\Omega)}^{2}+c_{1}\left\|\frac{\partial \rho}{\partial t}\right\|^{2} \leqslant c_{2}(\varepsilon)\left(\|\rho\|^{2}+\|\nabla u\|^{2}\right) .
\end{aligned}
$$

On the other hand we take $\eta=u$ in (2.40). Proceeding as above, we obtain

$$
\gamma \frac{d}{d t}\left(\frac{\partial u}{\partial t}, u\right)+c_{1}\|\nabla u\|^{2} \leqslant \gamma\left\|\frac{\partial u}{\partial t}\right\|^{2}+c_{2}\|\rho\|^{2} .
$$

We finally take $\eta=\frac{\partial u}{\partial t}$ in (2.40) and noting that $\left(\rho(\mathrm{CI}), \nabla \frac{\partial u}{\partial t}\right)=-\left((\mathrm{CI}) \nabla \rho, \frac{\partial u}{\partial t}\right)$

$$
\frac{d}{d t}\left(\gamma\left\|\frac{\partial u}{\partial t}\right\|^{2}+\frac{1}{2}\left(C\left(\nabla u+{ }^{t} \nabla u\right), \nabla u+{ }^{t} \nabla u\right)\right) \leqslant \tau\|\nabla \rho\|^{2}+c \tau^{-1}\left\|\frac{\partial u}{\partial t}\right\|^{2},
$$

for any strictly positive $\tau$.

We combine (2.45), $\delta(2.46)$ and (2.47) with a suitable $\tau$, and then obtain

$$
\begin{aligned}
& \frac{d}{d t}\left[\sigma\left\|\widetilde{B}^{1 / 2} \nabla B^{1 / 2} \nabla N^{-1} \rho\right\|^{2}+\sigma\|\rho\|_{-1}^{2}+\alpha\|\nabla \rho\|^{2}+e^{2} \mathrm{TR}(\mathrm{CI})\|\rho\|^{2}\right. \\
& \left.+\gamma \delta\left(\frac{\partial u}{\partial t}, u\right)+\gamma\left\|\frac{\partial u}{\partial t}\right\|^{2}+\frac{1}{2}\left(C\left(\nabla u+{ }^{t} \nabla u\right), \nabla u+{ }^{t} \nabla u\right)\right] \\
& +\|\rho\|_{H^{1}(\Omega)}^{2}+c_{1}\left\|\frac{\partial \rho}{\partial t}\right\|^{2} \leqslant c\left\|\frac{\partial u}{\partial t}\right\|^{2}+c\|\nabla \rho\|^{2}+c\|\nabla u\|^{2} .
\end{aligned}
$$

We now fix $\delta$ such that

$$
\gamma\left\|\frac{\partial u}{\partial t}\right\|^{2}+\frac{1}{2}\left(C\left(\nabla u+{ }^{t} \nabla u\right), \nabla u+{ }^{t} \nabla u\right)+\gamma \delta\left(\frac{\partial u}{\partial t}, u\right) \geqslant c_{2}\left(\left\|\frac{\partial u}{\partial t}\right\|^{2}+\|\nabla u\|^{2}\right),
$$

where $c_{2}>0$.

Setting

$$
\begin{aligned}
E_{3}(t)=\sigma\left\|\widetilde{B}^{1 / 2} \nabla B^{1 / 2} \nabla N^{-1} \rho\right\|^{2}+\sigma\|\rho\|_{-1}^{2}+\alpha\|\nabla \rho\|^{2}+e^{2} \mathrm{TR}(\mathrm{CI})\|\rho\|^{2} \\
\left.+\gamma \delta\left(\frac{\partial u}{\partial t}, u\right)+\gamma\left\|\frac{\partial u}{\partial t}\right\|^{2}+\frac{1}{2}\left(C\left(\nabla u+{ }^{t} \nabla u\right), \nabla u+{ }^{t} \nabla u\right)\right)
\end{aligned}
$$


we find

$$
E_{3}(t) \geqslant c_{1}\left(\left\|\frac{\partial u}{\partial t}\right\|^{2}+\|\nabla \rho\|^{2}+\|\nabla u\|^{2}\right)
$$

And therefore, we deduce an estimate of the form

$$
\frac{d E_{3}}{d t} \leqslant c E_{3}
$$

and then the uniqueness of $\rho_{\varepsilon}$ and $u_{\varepsilon}$. The uniqueness of $\mu_{\varepsilon}$ is deduced from (2.8).

THEOREM 2.1. Let the assumptions of Lemma 2.1 hold. Then, there exists a trio of functions $(\rho, \mu, u)$, solution of (1.3)-(1.5) such that $\rho \in L^{\infty}\left(0, T ; H_{\mathrm{per}}^{1}(\Omega)\right) \cap L^{2}(0, T$; $\left.H_{\mathrm{per}}^{2}(\Omega)\right) \cap \mathcal{C}\left([0, T] ; L^{2}(\Omega)\right), \mu \in L^{2}\left(0, T ; H_{\mathrm{per}}^{1}(\Omega)\right),|\rho| \leqslant 1$ almost everywhere in $\Omega_{T}$, $\frac{\partial \rho}{\partial t} \in L^{2}\left(\Omega_{T}\right), \phi(\rho) \in L^{2}\left(\Omega_{T}\right) ;$ and $u \in L^{\infty}\left(0, T ; \dot{H}_{\mathrm{per}}^{1}(\Omega)^{n}\right) \cap \mathcal{C}\left([0, T] ; \dot{L}^{2}(\Omega)^{n}\right)$, with $\frac{\partial u}{\partial t} \in L^{\infty}\left(0, T ; \dot{L}^{2}(\Omega)^{n}\right)$. Furthermore, we have the uniqueness of solution when $d=0$.

Proof: It follows from Lemma 2.1 that there exists a trio of functions $(\rho, \mu, u)$ and a subsequence $\left(\rho_{\varepsilon}, \mu_{\varepsilon}, u_{\varepsilon}\right)_{\varepsilon>0}$ (which we still denote by $\left.\left(\rho_{\varepsilon}, \mu_{\varepsilon}, u_{\varepsilon}\right)_{\varepsilon>0}\right)$ such that

$$
\begin{aligned}
& \rho_{\varepsilon}, \nabla \rho_{\varepsilon} \rightarrow \rho, \nabla \rho \quad \text { strongly in } L^{2}\left(\Omega_{T}\right) \text { and almost everywhere in } \Omega_{T} \text {, } \\
& \frac{\partial \rho_{\varepsilon}}{\partial t} \rightarrow \frac{\partial \rho}{\partial t} \quad \text { weakly in } L^{2}\left(\Omega_{T}\right) \text {, } \\
& \mu_{\varepsilon}, \nabla \mu_{\varepsilon} \rightarrow \mu, \nabla \mu \quad \text { weakly in } L^{2}\left(\Omega_{T}\right) \text {, } \\
& u_{\varepsilon}, \nabla u_{\varepsilon} \rightarrow u, \nabla u \quad \text { weakly-star in } L^{\infty}\left(0, T ; \dot{L}^{2}(\Omega)\right) \text {, } \\
& \frac{\partial u_{\varepsilon}}{\partial t} \rightarrow \frac{\partial u}{\partial t} \quad \text { weakly-star in } L^{\infty}\left(0, T ; \dot{L}^{2}(\Omega)\right), \\
& \phi_{\varepsilon}\left(\rho_{\varepsilon}\right) \rightarrow \phi(\rho) \quad \text { weakly in } L^{2}\left(\Omega_{T}\right) .
\end{aligned}
$$

Passing to the limit in the regularised problem, we find that $(\rho, \mu, u)$ is a solution of (1.3)-(1.5). The other points of the theorem also come from passing to the limit in the uniform estimates (2.27) and (2.37), as $\varepsilon$ goes to zero.

We don't succeed in obtaining the uniqueness of solution for (1.3)-(1.5) when $a \neq 0$ or/and $b \neq 0$, The difficulty appears in getting an estimate of the term $\left(\phi\left(\rho_{1}\right)-\phi\left(\rho_{2}\right), \frac{\partial \rho}{\partial t}\right)$ as we wanted. But, when we consider the case $d=0$, it is a simple matter to obtain the uniqueness of solution $(\rho, \mu, u)$. Indeed, we take $q=\rho, \eta=u$, $\eta=\frac{\partial u}{\partial t}$ in the corresponding non-regularised versions of (2.39) and (2.40) respectively. Combining estimates obtained as above, we get an estimate of the form (2.51), therefore the result.

\section{REFERENCES}

[1] J.W. Barrett and J.F. Blowey, 'An error bound for the finite element approximation of the Cahn-Hilliard equation with logarithmic free energy', Numer. Math. 72 (1995), 1-20. 
[2] A. Bonfoh and A. Miranville, 'On Cahn-Hilliard-Gurtin equations', Nonlinear Anal. 47 (2001), 3455-3466.

[3] J.W. Cahn, 'On spinodal decomposition', Acta Metall. 9 (1961), 795-801.

[4] J.W. Cahn and J.E. Hilliard, 'Free energy of a non-uniform system I. Interfacial free energy', J. Chem. Phys. 2 (1958), 258-267.

[5] M. Gurtin, 'Generalized Ginzburg-Landau and Cahn-Hilliard equations based on a microforce balance', Phys. D. 92 (1996), 178-192.

[6] J.L. Lions, Quelques méthodes de résolutions des problèmes aux limites non linéaires (Dunod, Paris, 1969).

[7] A. Miranville, 'Some generalizations of the Cahn-Hilliard equation', Asymptotic Anal. 22 (2000), 235-259.

[8] R. Temam, Infinite dimensional dynamical systems in mechanics and physics, (2nd Edition) (Springer-Verlag, Berlin, Heidelberg, New York, 1997).

University Kuala Lumpur

Malaysia France Institute

Sec 14, Jln, Teras Jernang

43650 Bdr Baru Bangi

Selangor D.E.

Malaysia 